\title{
Survey of on-road image projection with pixel light systems
}

Sadiq Rizvi, Marvin Knöchelmann, Peer-Phillip Ley, Roland Lachmayer

Sadiq Rizvi, Marvin Knöchelmann, Peer-Phillip Ley, Roland Lachmayer, "Survey of on-road image projection with pixel light systems," Proc. SPIE 10603, Photonics, Devices, and Systems VII, 1060314 (1 December 2017); doi: $10.1117 / 12.2292740$

SPIE. Event: Photonics Prague 2017, 2017, Prague, Czech Republic 


\title{
Survey of on-road image projection with pixel light systems
}

\author{
Sadiq Rizvi*a , Marvin Knöchelmann ${ }^{\mathrm{a}}$, Peer-Phillip Ley ${ }^{\mathrm{a}}$, Roland Lachmayer ${ }^{\mathrm{a}}$ \\ anstitute of Product Development, Leibniz University Hanover, Welfengarten 1A, 30167 Hanover
}

\begin{abstract}
HID, LED and laser-based high resolution automotive headlamps, as of late known as 'pixel light systems', are at the forefront of the developing technologies paving the way for autonomous driving. In addition to light distribution capabilities that outperform Adaptive Front Lighting and Matrix Beam systems, pixel light systems provide the possibility of image projection directly onto the street. The underlying objective is to improve the driving experience, in any given scenario, in terms of safety, comfort and interaction for all road users. The focus of this work is to conduct a short survey on this state-of-the-art image projection functionality.

A holistic research regarding the image projection functionality can be divided into three major categories: scenario selection, technological development and evaluation design. Consequently, the work presented in this paper is divided into three short studies. Section 1 provides a brief introduction to pixel light systems and a justification for the approach adopted for this study. Section 2 deals with the selection of scenarios (and driving maneuvers) where image projection can play a critical role. Section 3 discusses high power LED and LED array based prototypes that are currently under development. Section 4 demonstrates results from an experiment conducted to evaluate the illuminance of an image space projected using a pixel light system prototype developed at the Institute of Product Development (IPeG).
\end{abstract}

Findings from this work can help to identify and advance future research work relating to: further development of pixel light systems, scenario planning, examination of optimal light sources, behavioral response studies etc.

Keywords: pixel light systems, high resolution headlamps, image projection, accident analysis, illuminance

\section{INTRODUCTION}

\subsection{Pixel light systems}

Pixel light systems are able to address a 100,000+ pixels individually. Using camera information, this capability allows for a 'glare-free' distribution of light that is perfectly adapted to the needs of all road users. What really enables these systems to stand out however, is their image projection capability. This projection functionality may be used as an assistance feature during a lane change, for the projection of navigation data, warning signs, car status information etc. and for many other scenarios that remain to be investigated (See Figure 1).

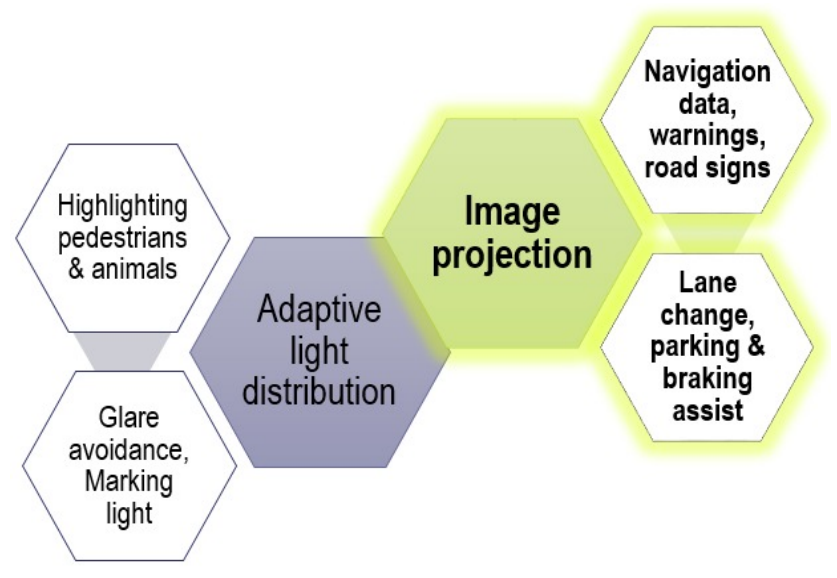

Figure 1. Functionalities offered by pixel light systems.

*rizvi@ipeg.uni-hannover.de; phone +49 511762 3503; fax +49 511762 4506; ipeg.uni-hannover.de 
As opposed to information being displayed on the vehicle dashboard or on a head up display (HUD) in the driver's field of view, pixel light systems can project information onto the street in front of the car at a distance of $10 \mathrm{~m}-25 \mathrm{~m}$. Whereas this makes the projections susceptible to distortions caused by environmental disturbances (stormy weather, uneven road etc.), it allows drivers to communicate with other road users in a road language that is truly evolutionary. This also means that the drivers have access to information without having to adjust sightline, and thus concentration, away from the road ${ }^{1}$. In the current state, pixel light systems can only be used in limited daylight or night time conditions.

\subsection{First image projection results from pixel light systems}

Pixel light system units using a digital micromirror device (DMD) and a HID lamp source have demonstrated ${ }^{2}$ on-road projections at a distance of up to $25 \mathrm{~m}$. Figure 2 (Left) shows a couple of scenario-based projections that can be possible with such a unit. DMD-based units allow parts of the light distribution to be modulated simultaneously. This classifies them, together with LCD and LED matrix, as an area based modulation technique. DMD-based units currently under development utilize high power LEDs and LED arrays as light sources. These are discussed in more detail in Section 3. A separate class of image projection technique consists of scanning technologies. Scanner resonant, Scanner partly resonant and Scanner quasi-static are instances of scanning technologies that are based on the modulation of light beams. ${ }^{1}$ Figure 2 (Right) shows working projection demonstrations from a laser scanning module ${ }^{3}$. A primary difference between the aforementioned two projection methods is that the DMD-based unit uses subtractive projection i.e. generation of dark pixels to project images/symbols, while the scanning module overlays the symbol onto a standard low or high beam. Whereas this limits the DMD-based unit to the projection of only greyscale images. Colored symbols may be overlaid onto low or high beam using the laser scanning module.
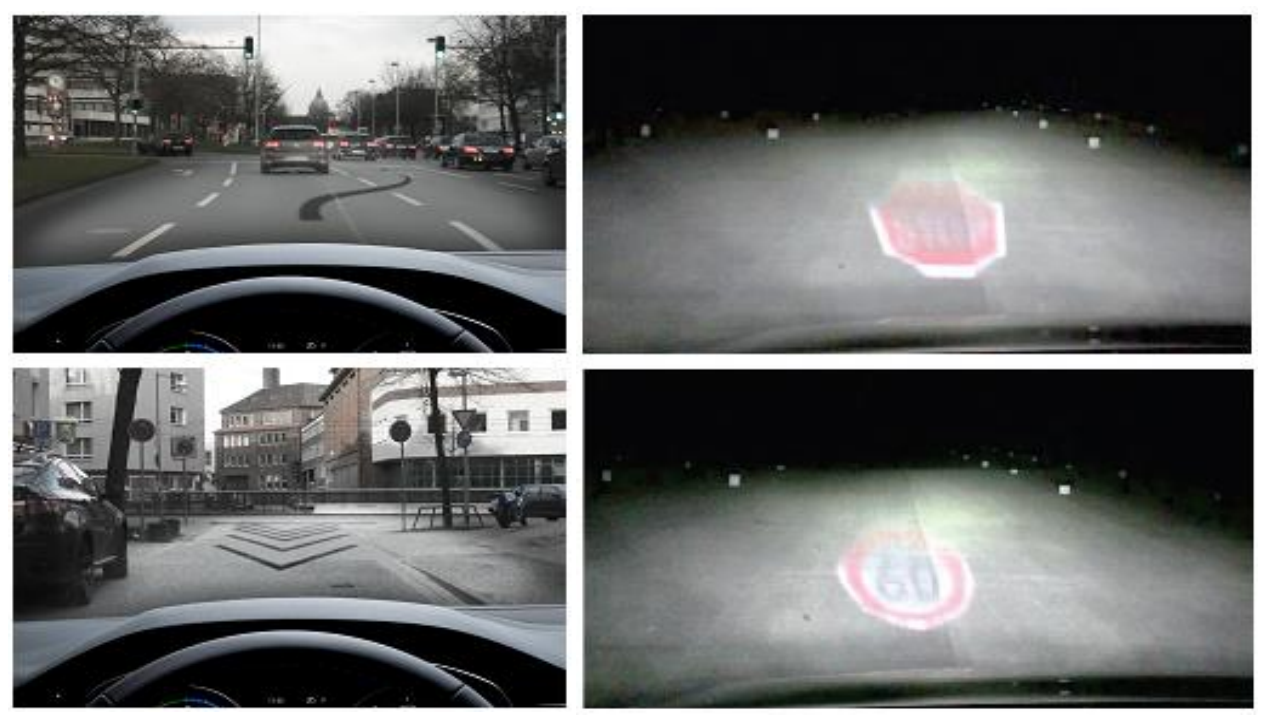

Figure 2. Left: Exemplary functionalities of DMD based automotive lighting units (Renderings) ${ }^{1}$. Right: Scanned working projections from a laser scanning module overlaid on low beam ${ }^{3}$.

\subsection{Humanocentric Projection}

Ergonomics is the scientific discipline concerned with the understanding of interactions among humans and other elements of a system. It is the profession that applies theory, principles, data and methods to design in order to optimize human wellbeing and overall system performance ${ }^{4}$. Further development of pixel light systems necessitates an ergonomic evaluation of context-based technological, physiological, psychological and other aspects. It follows that the ideal prototype will comply with all of the aforementioned 'Humanocentric' aspects.

Keeping in view the ever-growing need to conform to humanistic requirements, on an individual as well as a collective level, research regarding on-road image projection is best tackled by dividing this subject into three major categories: scenario selection, technological development and evaluation design. Although each area holds a distinct place, it is understood that all three areas are dependent on one another and so, must also evolve in tandem. This implies that research 
directions for one major area are related, to varying degrees, to directions in partner areas. Scenario selection forms the motivation and, essentially, the foundation of this work. A logical approach for selection is discussed in Section 2 with a couple of example scenarios. Whereby technological developments define and redefine the current limits/capabilities of the system, they can also be seen as a tool to solve new tasks. The study in Section 3 showcases prototypes currently under development. Evaluation design involves the design and implementation of studies/experiments to quantitatively and qualitatively rate system performance. Evaluations for on-road image projection can range anywhere from behavioral studies to studies that evaluate the photometric quality of the projected image space. Section 4 presents results from a short experiment conducted to rate the quality of the projected image space in terms of illuminance. These three research categories along with some respective example research directions are summarized in Figure 3.

\section{Scenario selection}

- Criteria selection (frequency of occurence, degree of casualty, time of day etc.)

-Driver, driver to driver, driver to pedestrian or bicyclist, driver to infrastructure etc.

- Selection of key driving maneuvers and traffic scenarios (lane change assistance, speed control, sharp curve, stormy conditions, traffic culture based scenarios, ambulance based scenarios etc.)

- Accident analysis (wheel slip, pedestrian hit, rear-end collisions etc.)

- Coordination with driving assistance systems (Front Assist, Cruise Control, Curve Speed etc.)

\section{Technological development}

-Efficient light sources (HID, LED, LED array, LD etc.)

-Possibilities for dynamic image projection, weather proofing etc. and compatibility to street topography, daylight and street lights etc.

-System integration into vehicles, enabling multi-technology platforms, man-machine interface, manufacturability

-Animal vision research

\section{Evaluation design}

-Evaluation of optimal photometric parameters (illuminance, contrast ratio, homogeneity, size etc.)

-Behavioral response studies of road users: response time, information overload, distraction, learnability, eye tracking, driver simulator etc.

-Assessment tools, information collection methods, inferential statistical analyses, comparative studies etc.

-Appropriateness of projected signs and symbols (cultural perceptions, color interpretation etc.)

Figure 3. Major areas of research for on-road image projection along with examples of respective research directions.

\section{SCENARIO SELECTION}

Survey of a number of research studies reveals that visual information forms the major portion of the received data for driving ${ }^{5}$. At night— - because of the paucity of light—human visual capacities are severely reduced, with a complete loss of the ability to see color and a dramatic loss in the ability to see fine spatial and temporal details. ${ }^{6}$ Whereas on one level, these human limitations lead to everyday misunderstandings, faulty assessments and obstructive traffic flow; more often than not, they lead to fatalities on the road ${ }^{7}$. A study ${ }^{8}$ analyzing data from the National Road Safety Council (KRBRD) for the year 2014 shows that although the likelihood of accident occurrence is higher during the day due to heavier traffic, the most fatal accidents still occur during night time. Since image projection using pixel light systems is a functionality designed to be used only in limited daylight or night time conditions, the total number of relevant driving maneuvers and traffic scenarios requiring analysis is already significantly reduced. Another notable study ${ }^{9}$ that examines the relation between lack of daylight and the number of fatal pedestrian accidents, demonstrates that the influence of light is scenario specific i.e. environmental lighting conditions do not always play a pivotal role in accidents. An overview of a number of 
similar studies indicates the requirement to scrutinize select late evening, night and early dawn time scenarios and accidents. The goal being to obtain a subset of driving maneuvers and traffic scenarios where the image projection functionality of pixel light systems makes a significant difference.

In order to understand the degree of (meaningful) impact image projection can make on the road, traffic scenarios and driving maneuvers need to be selected where pixel light systems are most likely to prove critical, if not only advantageous. Frequency of occurrence and degree of casualty are examples of quantitative criteria that give priority to some scenarios over others. Likewise, interaction between road users is an example of a qualitative criterion that can be enhanced in select traffic scenarios via use of projections. Better interaction translates into fewer misunderstandings and improved traffic flow. Thus, such a criterion qualifies as a better candidate compared to a luxury-centric criterion (greeting messages etc.) that solely improves driver comfort. The approach taken here can be correlated to the conception of worst-case scenarios in risk management. This is a common practice in strategic and scenario planning ${ }^{10}$ and one that usually yields solutions for simpler cases as by-products of the process. Thus an algorithm centered on the pixel light system delivering a humanocentric projection takes form here (See Figure 4). Notice the larger space attributed to the technological research aspect. This is because technological research and development is (at least prevalently) seen to be the driving factor.

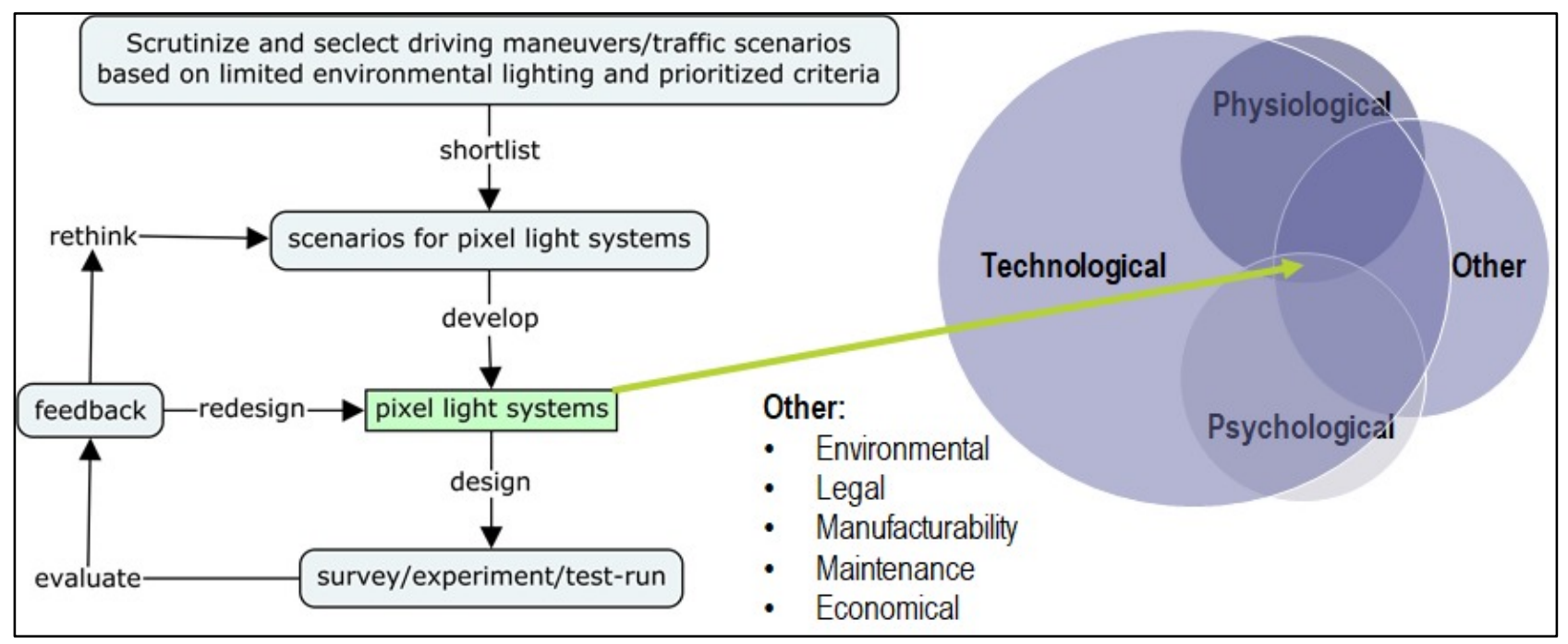

Figure 4. Process flow for developing humanocentric pixel light systems.

In a recent and comprehensive study ${ }^{11}$; a thorough analysis of accident data provided by GIDAS (German In-Depth Accident Study) and DESTATIS (Federal statistical office) for limited daylight conditions, for the Hanover and Dresden regions and for a period spanning 1995-2013 confirms that the most fatal accidents occur at night and on highways. Misjudgment of road speed due to road conditions and curving is quoted as the major cause for losing control of the vehicle. Other prevalent causes for accident include: insufficient distance to vehicle ahead, lack of attention to traffic from rear, poor visibility and wrong lane use. Some scenarios developed to counter such accidents using on-road image projections provided in the study are shown in Figure 5.
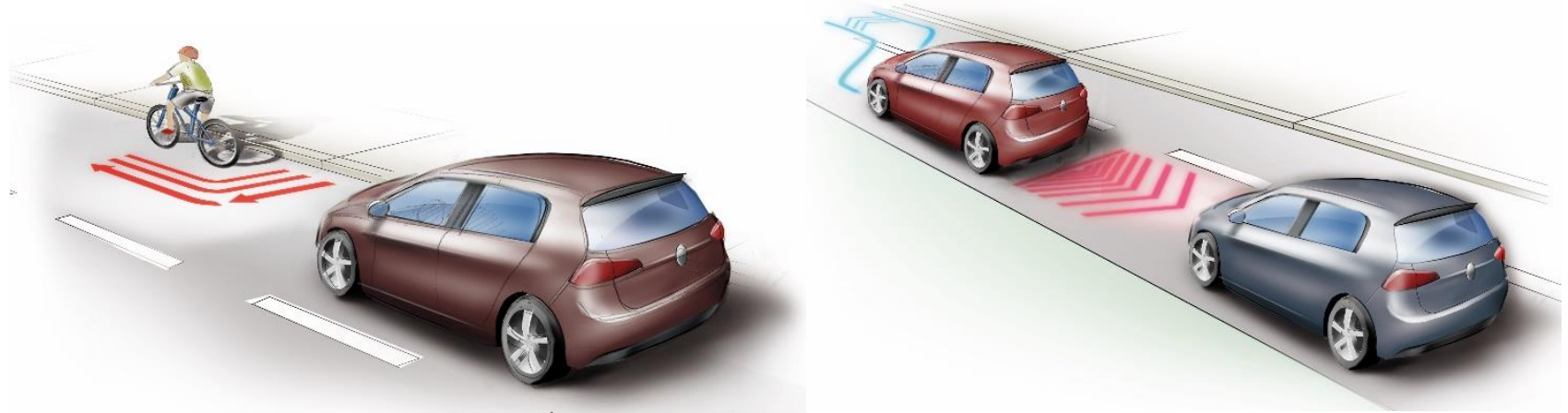

Figure 5. Some example scenarios showing dynamic on-road image projections for accident prevention. 


\section{TECHNOLOGICAL DEVELOPMENT}

As already mentioned in Section 1.3, technological research and development plays a leading role in the further advancement of light-based on-road image projection systems. With exception to technological innovations that take place purely by accident, technological research is primarily motivation based. In the context of this study, this means that the pixel light system or any subsystem within it can be developed to: mitigate danger in a certain scenario, autonomously identify scenarios that require image projections, reduce the thermal load on the light source etc. These are all valid examples of motivations driving technological development. For the purposes of this study, the motivation exemplified concerns with the use of more efficient light sources. LED based light sources offer directional light, are more efficient, compact and long lasting ${ }^{12}$. These are all benefits that help to improve the quality of the projected image. A description of the current pixel light system assembly is provided followed by a note on the viability of an LED based pixel light system.

\subsection{Description of the current pixel light system assembly}

Design of the current DMD-based pixel light system prototype developed at IPeG makes use of typical video projector components with the exception of the colour wheel. There is no use for colour wheels since headlamps are currently allowed to illuminate the road using only white light. The system comprises of a light source, an integrator rod, relay optics, a TIR-Prism, a DMD and projection optics (See Figure 6).

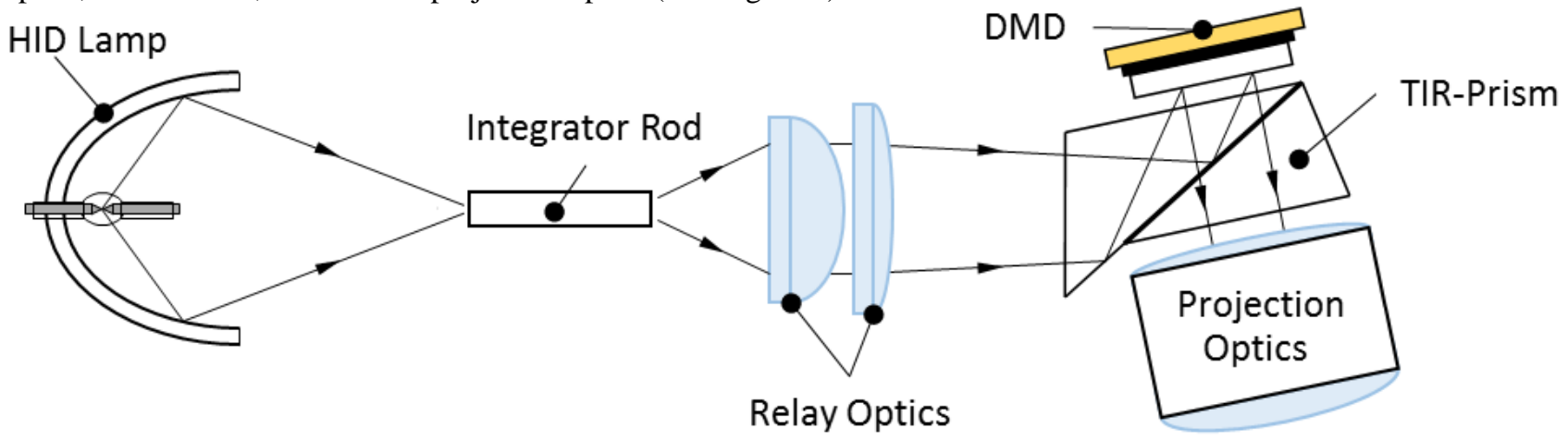

Figure 6. Simplified pixel light system assembly ${ }^{13}$.

If a high intensity discharge lamp is used as a light source, an elliptical reflector is necessary to collect and focus the light from the lamp onto a small point on the integrator rod. The integrator rod is installed to obtain a more uniform and flat spatial distribution of the light on the projection surface. For an adequate magnification of the projection, the numerical aperture of the integrator rod and the projection must match. Relay optics are used here for this purpose. The TIR-Prism separates the light into a projection path and an illumination path. The former is directed towards the DMD. A DMD consists of an array of millions of micromirrors that can be addressed individually. Each micromirror has three positions and represents a pixel. In the "on-state" the incident light is being reflected onto the projection optics, whereas in the "offstate" the light is being deflected onto an absorber (not displayed in the figure). The so-called "flat-state" practically only exists when the DMD is not powered.

\subsection{LED-based DMD pixel light systems}

For an efficient DMD-based headlamp system, various components used in a video projector are inefficient and therefore dispensable. With regards to efficiency, the light source offers the highest potential to be improved. Currently, LED headlights seem to be the future in automotive lighting. Compared to a halogen lamp $(27 \mathrm{~lm} / \mathrm{W})$ or a xenon lamp $(90 \mathrm{~lm} / \mathrm{W})$ the luminance efficacy up to $140 \mathrm{~lm} / \mathrm{W}$ for a white LED is distinctly higher. In addition to that, LEDs offer a higher durability, they are mercury-free and have low power consumption. Typically a single white-LED package has a power consumption of $\sim 15.2 \mathrm{~W}$ meaning $\sim 2125$ lumens. To achieve the same luminous flux with a xenon lamp, the power consumption required is $23.6 \mathrm{~W}$ i.e. $~ 55 \%$ increase in power consumption ${ }^{12}$. LEDs do not require IR, UV, and dichroic filters, and collection of light from LEDs is more efficient than from thermal light sources. The primary issue with using LEDs is coupling their output efficiently into small étendue systems, like DLP or any microdisplay ${ }^{13}$. However, workarounds to this issue are already under way. With this short overview, it is clear that a LED-based pixel light system can potentially be a more efficient alternative to a lamp-based system. 


\section{EVALUATION DESIGN}

\subsection{Photometric evaluation of the projected image space}

Visual performance and traffic safety are highly correlated to the amount and quality of visual information we can obtain from the road, its immediate environment ${ }^{14}$ and, in this case, from the on-road projected image. One area of concern is the development of image projection techniques that are adaptive to dynamic scenarios, adverse weather conditions and rough street topologies. The other, more core, challenge is to work on the projected image space itself. The goal here is to project the desired image on the street such that:

1. The projected image causes minimal obstruction to the visual information already being received.

2. The projections are suitably positioned with high enough resolution etc. to ensure effortless comprehension.

\subsection{Experiment to evaluate illuminance}

A basic static experiment is designed to evaluate the illuminance of the projected image space. The pixel light system prototype and projection optics developed at IPeG are used for this purpose. Two of these projectors, tilted $-1 \%$ as per ECE regulations, are mounted in front of the car at a height of $880 \mathrm{~mm}$ and a distance of $1440 \mathrm{~mm}$ apart. The mounted system (Left) and a sketch of the road scene of the experimental setup (Right) are shown in Figure 7.
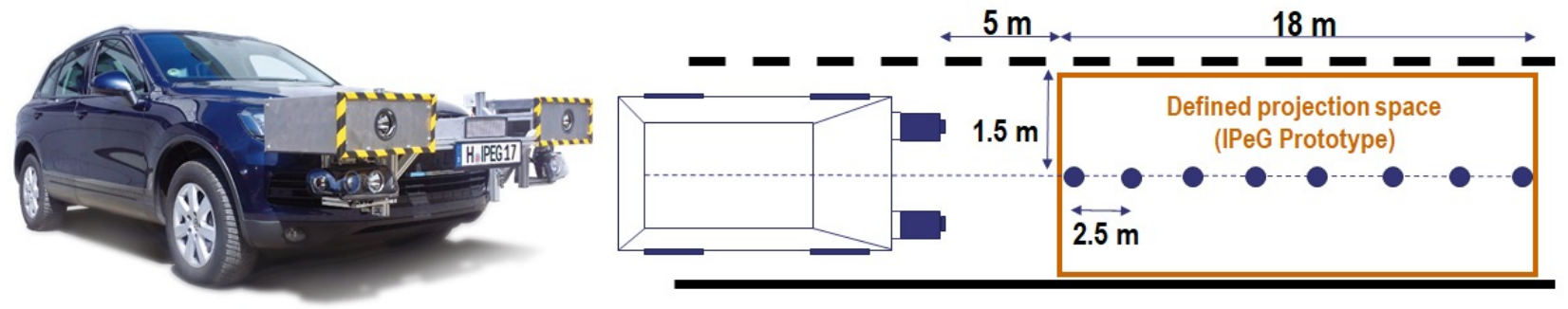

Figure 7. Left: Pixel light system prototype developed at IPeG mounted onto a car, Right: Bird's eye view of the experimental setup to measure illuminance of the on-road image projection

For the evaluation, illuminance values from the projected image space measured using a Luxmeter outdoors are compared to illuminance values measured, for the same system, using a Gonio-photometer in laboratory conditions. Illuminance is defined as the amount of light that covers a particular surface and is measured in units of Lux (lx). For the outdoors measurement, at night time- a total of 8 spots are marked on a dark and dry asphalt road devoid of street lights. The spots are positioned linearly and at equal spaces along the central axis of the projection space as shown in Figure 8 (Right). For a duplicate setup in the laboratory, illuminance values are measured with the help of a Gonio-photometer and simulated at a $-1 \%$ tilt angle in LucidShape ${ }^{\circledR}$. See Bird's eye view of the results in Figure 8 (Left). Illuminance values from outdoors (dots) and laboratory (curve) are plotted and found to match to an acceptable degree of accuracy (See Figure 8). In addition, the projection space of the laboratory test is compared to a DMD headlamp road-test ${ }^{15}$ meeting photometry regulations. Here it is found that the range of image projection space projected by the IPeG Prototype outreaches the other headlamp's space by a significant area. The aforementioned illuminance evaluations emphasize the repeatability of laboratory results outdoors and highlight a key advantage of the IPeG prototype versus a previously developed DMD headlamp.
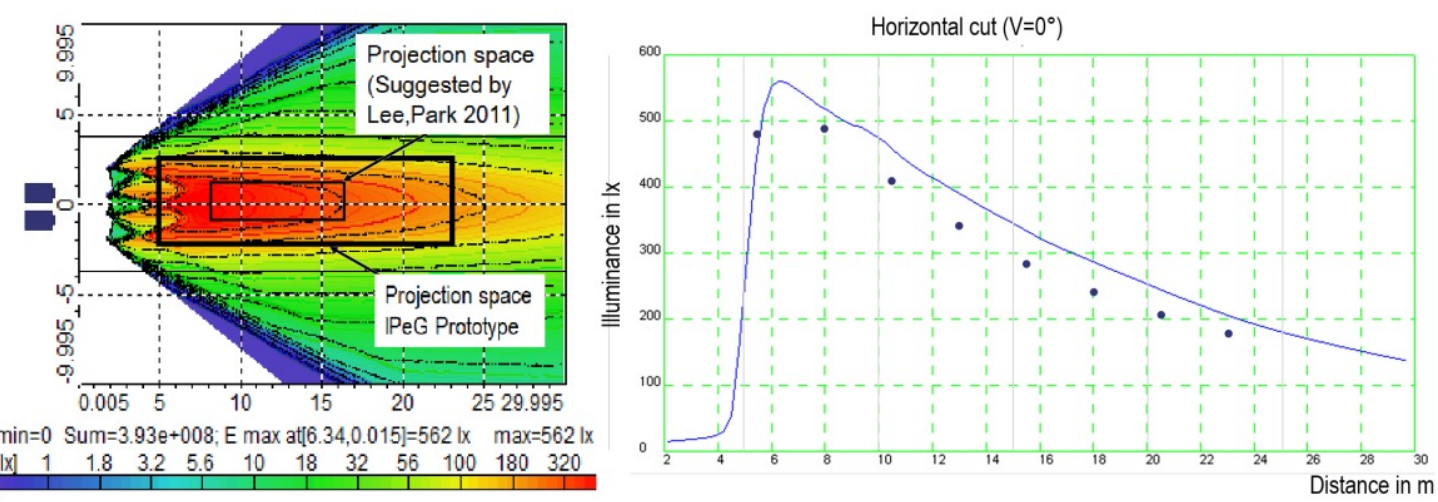

Figure 8. Left: Simulation of lab results in LucidShape ${ }^{\circledR}$, Right: Illuminance measurements Outdoor (dots) vs. lab (curve) 
Some of the important next steps in the evaluation of on-road image projections should take into account: dynamic driving scenarios, contrast ratio of the image projection space, passenger-seat or pedestrian perceptions, human response study etc.

\section{CONCLUSIONS}

A holistic approach to the discussion regarding on-road image projections using pixel light systems is presented. The topic is broken into three major categories, namely: scenario selection, technological development and evaluation design. The included short studies explore example research directions whilst serving as a means to elaborate on the suggested approach. It is hoped that findings from this work can help to identify and advance relevant future research work, especially with regards to: compilation of key driving scenarios, development of LED-based pixel light systems and evaluation of the image projection space.

\section{ACKNOWLEDGEMENTS}

This project was funded by the Lower Saxony Ministry of Science and Culture within the framework of the PhD-program 'Tailored Light'. I would like to extend thanks to my colleagues and supervisor at IPeG for their valuable input, especially the workshop staff for helping install the pixel light system onto the test car and to Bastian Möller for his designer expertise (Figure 5).

\section{REFERENCES}

[1] Kloppenburg, G., Wolf, A., Lachmayer, R., "High-resolution vehicle headlamps: technologies and scanning prototype," Advanced Optical Technologies 5(2), 147-155 (2016).

[2] A. Wolf, G. Kloppenburg, R. Danov, R. Lachmayer, "DMD Based Automotive Lighting Unit," in DGaO Proceedings 2016.

[3] J. Roth, J. Wallaschek, G. Kloppenburg, R. Lachmayer, B. Meyer, S. Thomschke, "RGB-Laser Scanning Module for Onroad Projection," in Khanh (Hg.) 2015 - Proceedings of the 11th International, pp. 385-394.

[4] "What is Ergonomics?", Definition and Domains of Ergonomics | IEA Website, <http://www.iea.cc/whats/index.html> (accessed 1 July 2017).

[5] T. Q. Khanh and W. Huhn, "Sichtverbesserungssysteme," Handbuch Fahrerassistenzsysteme, 448-470 (2012) [doi:10.1007/978-3-8348-8619-4_31].

[6] E. Warrant, "Superior vision in nocturnal insects inspires new night vision technologies," SPIE Newsroom (2016) [doi:10.1117/2.1201603.006426].

[7] J. Locher and S. Voelker, "The Influence of Vehicle Beam Patterns on Safety and Acceptance," SAE Technical Paper Series (2004) [doi:10.4271/2004-01-1766].

[8] P. Aleksandrowicz, Ł. Muślewski, and M. Lewalski, "Visibility Of An Untypical Obstacle In Low Beam Headlights During Driving At Night," Journal of KONES. Powertrain and Transport 23(1), 17-24 (2016) [doi:10.5604/12314005.1213505].

[9] B. Wördenweber, J. Wallaschek, P. Boyce and D. D. Hoffman, Automotive Lighting and Human Vision, Springer Berlin, Berlin (2007).

[10] "Worst-case scenario," Wikipedia, 22 June 2017, <https://en.wikipedia.org/wiki/Worst-case_scenario> (accessed 3 July 2017).

[11]Krahnstöver, A. Z., Licht führt!? Konzeption und Evaluation von Fahrmanöverunterstützung durch lichtbasierte Fahrerassistenzsysteme, Springer Fachmedien Wiesbaden GmbH, Wiesbaden (2017).

[12] Bielawny, A., Schupp, T., Neumann, C., "Automotive Lighting Continues to Evolve," Optics and Photonics News 27(11), 36 (2016).

[13]“Application Report: DLPTM System Optics (July 2010).,” rep., Texas Instruments, 1-26.

[14]W. Adrian, "Visibility of targets: Model for calculation," Lighting Research \& Technology 21(4), 181-188 (1989) [doi:10.1177/096032718902100404].

[15]T. Lee, G. Park, H. Kim, I. Park, K. Lee, B. Ahn, "The Development of the Automotive Headlamp using the DMD,” in Khanh (Hg.) 2011 - Proceedings of the 9th International, pp. 524-534. 